\title{
MANY DISJOINT TRIANGLES IN CO-TRIANGLE-FREE GRAPHS
}

\author{
MYKHAYLO TYOMKYN
}

\begin{abstract}
We prove that any $n$-vertex graph whose complement is triangle-free contains $n^{2} / 12-$ $o\left(n^{2}\right)$ edge-disjoint triangles. This is tight for the disjoint union of two cliques of order $n / 2$. We also prove a corresponding stability theorem, that all large graphs attaining the above bound are close to being bipartite. Our results answer a question of Alon and Linial, and make progress on a conjecture of Erdős.
\end{abstract}

\section{INTRODUCTION}

One of the classical results in extremal graph theory, Goodman's theorem [4, states that in every 2-colouring of the edges of the complete graph $K_{n}$ the number of monochromatic triangles is at least $\frac{1}{4}\left(\begin{array}{l}n \\ 3\end{array}\right)-o\left(n^{3}\right)$, that is, about a quarter of all possible triangles are guaranteed to be monochromatic. With this in mind, Erdős [2, 3] asked about the number of edge-disjoint monochromatic triangles in any 2-colouring of $K_{n}$.

To be more formal, a triangle packing of a graph $G$ is a collection of edge-disjoint triangles in $G$. The size of a triangle-packing is the total number of edges it contains 1 Define $f(n)$ to be the largest number $m$, such that every 2-colouring of the edges of $K_{n}$ contains a triangle packing of size $m$, in which each triangle is monochromatic.

As a basic example, consider $n=6$. By the folklore fact about Ramsey numbers, any 2-colouring of $K_{6}$ contains a monochromatic triangle, and it is not hard to see that it has to contain at least two such triangles. However, they need not be edge-disjoint, as can be seen by taking a 5-cycle and replicating a vertex. So, $f(6)=3$.

In general, the obvious upper bound of $f(n) \leq n^{2} / 4-o\left(n^{2}\right)$ is seen to hold by considering the balanced complete bipartite graph and its complement. Erdös [2, 3] conjectured that this is tight 2

\section{Conjecture 1.1.}

$$
f(n)=\frac{n^{2}}{4}-o\left(n^{2}\right)
$$

To draw a parallel to Goodman's theorem, Conjecture 1.1 states that every 2-edge-colouring of $K_{n}$ admits a packing with monochromatic triangles, containing about one half of all possible edges.

In previous works, Erdős, Faudree, Gould, Jacobson and Lehel [3] proved a first non-trivial lower bound of $f(n) \geq(9 / 55) n^{2}+o\left(n^{2}\right)$. Keevash and Sudakov [6] improved this, by using the fractional relaxation of the problem, to $f(n) \geq n^{2} / 4.3+o\left(n^{2}\right)$. Alon and Linial (see [6]) suggested, as a step towards Conjecture 1.1, to consider the natural class of colourings, in which one of the colour classes is triangle-free.

At this stage it will be more convenient to break the symmetry and speak of a graph and its complement. A graph is said to be co-triangle-free if its complement is triangle-free. Equivalently, co-triangle-free graphs are graphs with independence number at most 2. Define $g(n)$ to be the

\footnotetext{
Date: January 9, 2020.

${ }^{1}$ This is obviously the number of the triangles in the packing times 3 . We prefer the present scaling for technical and presentation reasons.

${ }^{2}$ In [2, 3, 6] the $n^{2} / 4+o\left(n^{2}\right)$ notation is used. It is understood that the additive $o\left(n^{2}\right)$-term can be negative, as this is the case e.g. in the above example. Hence, we believe the expression $n^{2} / 4-o\left(n^{2}\right)$ better reflects the nature of the conjecture.
} 
largest number $m$, such that every co-triangle-free graph on $n$ vertices contains a triangle packing of size $m$. The same example as for $f(n)$ - the disjoint union of two cliques of order $n / 2$, shows that $g(n) \leq n^{2} / 4-o\left(n^{2}\right)$, and Conjecture 1.1 would imply that this is tight.

Conjecture 1.2.

$$
g(n)=\frac{n^{2}}{4}-o\left(n^{2}\right)
$$

Yuster [7] worked specifically on Conjecture 1.2, and proved that any potential counterexample to it must have between $0.2501 n^{2}$ and $3 n^{2} / 8$ edges. That is, its size cannot be too close to, or too far from the Mantel threshold.

Our aim in this note is to give a short proof of Conjecture 1.2 .

Theorem 1.3. We have

$$
g(n)=\frac{n^{2}}{4}-o\left(n^{2}\right)
$$

Moreover, we classify the extremal graphs. An $n$-vertex graph is said to be $\varepsilon$-far from being bipartite if at least $\varepsilon n^{2}$ edge deletions are required in order to make it bipartite.

Theorem 1.4. For every $\varepsilon>0$ there exists $\delta>0$ such that any co-triangle-free graph $G$ of order $n$, whose complement is $\varepsilon$-far from being bipartite, has a triangle packing of size $(1 / 4+\delta) n^{2}+o\left(n^{2}\right)$.

In other words, every co-triangle free graph on $n$ vertices admits a triangle packing on $n^{2} / 4-o\left(n^{2}\right)$ edges, and the graphs achieving at most $n^{2} / 4+o\left(n^{2}\right)$ are essentially co-bipartite.

At the core of our proof is Lemma 3.2, which states that if a large graph $G$ is 'critical', that is its complement $\bar{G}$ is triangle-free, not bipartite, but can be made bipartite by deleting a vertex, then $G$ has a fractional triangle packing of size larger than $n(n-1) / 4$. This, combined with the integerfractional transference principle of Haxell and Rödl (Proposition 2.1), averaging over fractional packings, and a computer verification for small values of $n$ in the spirit of [6], yields the proof of Theorem 1.3 .

To prove Theorem 1.4, in addition to the above tools, we apply a theorem of Alon, Shapira and Sudakov (Proposition 4.1) on the structure of graphs with a large edit distance to a monotone graph property.

The rest of the paper is organized as follows. In Section 2 we will collect some known facts about fractional and integer triangle packings. The proofs of the crucial Lemma 3.2, and of Theorem 1.3 are carried out in Section [3. In Section 4 we derive Theorem 1.4, and in Section 5 we discuss Conjecture 1.1 and related open questions.

\section{Preliminaries}

Denote by $\nu(G)$ the size of the largest triangle packing in $G$. In this notation,

$$
g(n)=\min \{\nu(G):|G|=n, G \text { is co-triangle-free }\} .
$$

A fractional triangle packing of $G$ is a function $w$ from $\mathcal{T}(G)$, the set of all triangles in $G$, to $[0,1]$ such that every edge $e \in E(G)$ satisfies $\sum_{T \in \mathcal{T}(G): e \subset T} w(T) \leq 1$. The size of a fractional packing

is given by $3 \sum_{T \in \mathcal{T}(G)} w(T)$. Define $\nu^{*}(G)$ to be the maximum size of a fractional triangle packing of $G$; by compactness, this is well-defined. Note that ordinary triangle packings are precisely the integer-valued fractional packings - indeed, determining $\nu^{*}(G)$ is the LP-relaxation of the integer linear program of finding $\nu(G)$, so that $\nu^{*}(G) \geq \nu(G)$ for every graph $G$. Consequently, we define the function $g^{*}(n)$ to be the fractional counterpart to $g(n)$,

$$
g^{*}(n):=\min \left\{\nu^{*}(G):|G|=n, G \text { is co-triangle-free }\right\} .
$$

By the above, this function satisfies $g^{*}(n) \geq g(n)$ for every $n$. On the other hand, as a consequence of the seminal theorem of Haxell and Rödl [5], $\nu(G) \geq \nu^{*}(G)-o\left(n^{2}\right)$ holds for every $n$-vertex graph $G$. Therefore, we have 


\section{Proposition 2.1.}

$$
g(n) \geq g^{*}(n)-o\left(n^{2}\right)
$$

By virtue of Proposition 2.1 we can work with fractional instead of integer triangle packings at virtually no loss. Hence, going forward, the term "packing", unless specified otherwise, will refer to fractional triangle packings. For an $n$-vertex co-triangle-free graph $G$ define the packing density of $G$, to be

$$
\eta(G):=\frac{\nu^{*}(G)}{n(n-1)}
$$

It is well-known that packing densities are monotone under averaging (see e.g. Lemma 2.1 in [6]).

Lemma 2.2. Suppose that $G$ is a graph on $n$ vertices, and let $G_{1}, \ldots, G_{n}$ be its induced subgraphs of order $n-1$. Then

$$
\eta(G) \geq \frac{1}{n} \sum_{i=1}^{n} \eta\left(G_{i}\right)
$$

Proof. Without loss of generality, assume that $V(G)=[n]$, and $V\left(G_{i}\right)=[n] \backslash\{i\}$. Let $w_{i}$ be a packing of $G_{i}$ of size $\nu^{*}\left(G_{i}\right)$. Consider $w=\frac{1}{n-2} \sum w_{i}$, which is a function on $\mathcal{T}(G)$. Any given edge $\{i, j\}$ contributes 0 to $w_{i}+w_{j}$, so it receives a total weight of

$$
\frac{1}{n-2} \sum_{k \neq i, j} \sum_{T=\{i, j, \ell\} \in \mathcal{T}\left(G_{k}\right)} w_{k}(T) \leq \frac{1}{n-2} \sum_{k \neq i, j} 1=1 .
$$

Thus, $w$ is a packing of $G$ of size $\frac{1}{n-2} \sum_{i=1}^{n} \nu^{*}\left(G_{i}\right)$, which implies

$$
n(n-1) \eta(G)=\nu^{*}(G) \geq \frac{1}{n-2} \sum_{i=1}^{n} \nu^{*}\left(G_{i}\right)=(n-1) \sum_{i=1}^{n} \eta\left(G_{i}\right),
$$

and the desired inequality follows.

Corollary 2.3. With the above notation,

$$
\eta(G) \geq \min _{1 \leq i \leq n} \eta\left(G_{i}\right)
$$

We say that $G$ is co-bipartite if its complement is bipartite. Equivalently, $G$ is co-bipartite if $V(G)$ is spanned by a disjoint union of two cliques; clearly, co-bipartite graphs are co-triangle-free. We shall need the following straightforward bound on packings of co-bipartite graphs.

Lemma 2.4. For any co-bipartite $G$ of order $n \geq 6$ we have

$$
\nu^{*}(G) \geq \frac{n(n-2)}{4} .
$$

Proof. $G$ contains two disjoint cliques of sizes $a$ and $n-a$, for some $0 \leq a \leq n / 2$. Since each clique of order $m \geq 3$ admits a packing of size $\left(\begin{array}{c}m \\ 2\end{array}\right)$, by convexity of the binomial coefficients, we have

$$
\nu^{*}(G) \geq\left(\begin{array}{l}
a \\
2
\end{array}\right)+\left(\begin{array}{c}
n-a \\
2
\end{array}\right) \geq 2\left(\begin{array}{c}
n / 2 \\
2
\end{array}\right)=\frac{n(n-2)}{4} .
$$

A fractional triangle decomposition of $G$ is a packing, in which $\sum_{T \in \mathcal{T}(G): e \subset T} w(T)=1$ holds for every edge $e \in E(G)$. Fractional decompositions are packings of the largest possible size $e(G)$.

Lemma 2.5. Suppose that $G$ is a graph on $n$ vertices, and let $G_{1}, \ldots, G_{n}$ be its induced subgraphs of order $n-1$. If each $G_{i}$ has a fractional triangle decomposition, then so does $G$.

Proof. Assuming $V(G)=[n]$, and $V\left(G_{i}\right)=[n] \backslash\{i\}$, define $w$ as in the proof of Lemma 2.2, We obtain (2.1) with equality in place of the inequality. Thus, $w$ is a fractional decomposition of $G$. 
Let $K_{n}^{-k}$ denote the graph obtained from $K_{n}$ by removing a $k$-edge matching.

Lemma 2.6. For all integers $n \geq 7$ and $0 \leq k \leq\lfloor n / 2\rfloor$ the graph $K_{n}^{-k}$ has a fractional triangle decomposition.

Proof. It is easy to check by hand that this holds for $n=7$. The rest follows by induction, applying Lemma 2.5.

\section{Proof of Theorem 1.3}

Theorem 1.3 follows readily from the following stability result.

Lemma 3.1. Suppose that $G$ is co-triangle-free, with $|G| \geq 26$ and $\eta(G) \leq 1 / 4$. Then $G$ is cobipartite.

The reason for the threshold of 26 is that for $|G| \leq 25$, the 'natural enemy' of bipartite graphs in our problem, namely the blow-up of the 5-cycle, achieves $\eta \leq 1 / 4$. This, however, happens only for small $n$ : at $n=25$, the 5-blow-up of $C_{5}$ attains precisely $\eta=1 / 4$, and for larger $n$, as Lemma 3.1 claims, only co-bipartite graphs achieve packing densities of at most $1 / 4$.

Let us first show that Theorem 1.3 is indeed implied by Lemma 3.1

Proof of Theorem 1.3. The complement of $K_{n / 2, n / 2}$ certifies that $g(n) \leq n^{2} / 4-o\left(n^{2}\right)$. To see the other direction, suppose for a contradiction that $g(n) \leq n^{2} / 4-\Omega\left(n^{2}\right)$. Then, by Proposition 2.1 , we have

$$
g^{*}(n) \leq \frac{n^{2}}{4}-\Omega\left(n^{2}\right)
$$

This means, there exists $\varepsilon>0$ such that for large $n$ there is a co-triangle-free $G$ with $n$ vertices and $\eta(G)<1 / 4-\varepsilon$. By Lemma 3.1, $G$ is co-bipartite. However, in this case, by Lemma 2.4,

$$
\nu^{*}(G) \geq \frac{n(n-2)}{4},
$$

so $\eta(G) \geq 1 / 4-O(1 / n)$, contradicting $\eta(G)<1 / 4-\varepsilon$. Hence,

$$
g(n)=\frac{n^{2}}{4}-o\left(n^{2}\right)
$$

The proof of Lemma 3.1 is carried out by induction on $n$. For both the induction base $(n=26)$ and the step we require the following crucial lemma. Call a co-triangle-free graph $G$ critical if $G$ is not co-bipartite, but contains a vertex whose removal will make it co-bipartite.

Lemma 3.2. Every critical graph $G$ with $|G|=n \geq 18$ satisfies

$$
\nu^{*}(G) \geq \frac{n^{2}-17}{4}>\frac{n(n-1)}{4} .
$$

In particular,

$$
\eta(G)>\frac{1}{4}
$$

Before giving the proof of Lemma 3.2, let us show how it implies Lemma 3.1

Proof of Lemma 3.1. We proceed by induction on $n$. The statement for $n=26$ has been computer verified via the following algorithm (the program and the execution logs are provided in supplemental files to this paper). Our code is a modification of the code from the paper of Keevash and Sudakov [6], tailored to meet the specific requirements of our proof.

Initialization: create the list $L_{n}$ of all triangle-free graphs on $n=6$ vertices, and calculate $\nu^{*}$ for their complements. 
Iteration: For each $n \geq 7$, go through all one-vertex triangle-free extensions of the graphs in $L_{n-1}$, and select from them the graphs $H$ with $\eta(\bar{H}) \leq 1 / 4$, to form the list $L_{n}$. By Corollary 2.3, any other triangle-free graph $G$ of order $n$ must have $\eta(\bar{G})>1 / 4$. If $L_{n}$ is empty, the algorithm terminates. Otherwise, move to the next iteration step.

At $n=17$, before proceeding with the iteration, delete from $L_{17}$ all bipartite graphs (be aware that this is a one-off action, which is carried out only at $n=17)$. After that, perform the iteration step for $n=18$, and continue as previously. By Lemma 3.2 and Corollary 2.3, for $n \geq 18$ every co-triangle-free $n$-vertex graph $G$ with $\chi(\bar{G})>2$ and $\eta(G) \leq 1 / 4$ is a one-vertex extension of an $(n-1)$-vertex graph with the same properties. Therefore, for each $n \geq 18$ the list $L_{n}$ will contain precisely all triangle-free, non-bipartite $n$-vertex graphs $H$ satisfying $\eta(\bar{H}) \leq 1 / 4$.

Termination: The algorithm terminates if for some $n$ the list $L_{n}$ is empty.

Outcome: The program run terminates at $n=26$, when $L_{26}$ turns out to be empty. In fact, at $n=25$ the single graph in $L_{n}$, up to isomorphism, is the 5-blowup of $C_{5}$, and it has no valid extensions to $n=26$. This completes the proof of the induction base.

To see that Lemma 3.2 also implies the induction step for Lemma 3.1, let $G$ be as in Lemma 3.1. with $|G|=n \geq 27$, and let $G_{1}, \ldots, G_{n}$ be the induced subgraphs of $G$ of order $n-1$. By Corollary 2.3, we have $\eta\left(G_{i}\right) \leq 1 / 4$ for some $i$, and note that $G_{i}$ is co-triangle-free. By the induction hypothesis, $G_{i}$ is co-bipartite. If $G$ is co-bipartite, we are done. Otherwise, $G$ is critical, so, by Lemma 3.2, we have $\eta(G)>1 / 4$, a contradiction.

Remark 3.3. Strictly speaking, the proof of Lemma 3.1 uses Lemma 3.2 only for $n \geq 27$. The latter was stated and proved for $n \geq 18$ for the purpose of accelerating the computer search needed to prove Lemma 3.1 for $n=26$.

Proof of Lemma 3.2. Suppose that $n \geq 18$, and $G$ is a critical graph on $n$ vertices. Then there exists a vertex $v \in G$ such that $G^{\prime}:=\overline{G \backslash\{v\}}$ is bipartite. Let $U \bullet W$ be a bipartition of $V\left(G^{\prime}\right)$, that is $G^{\prime}=G^{\prime}[U, W]$, and note that the graphs $G[U]$ and $G[W]$ are complete. Note also that we can assume

$$
\min \{|U|,|W|\} \geq 7
$$

as otherwise $G[U] \cup G[W]$ would contain a packing of size more than $n(n-1) / 4$, and we would be done. Define

$$
\begin{aligned}
& A:=N_{G}(v) \cap U, \\
& B:=N_{G}(v) \cap W, \\
& X:=U \backslash A=N_{\bar{G}}(v) \cap U, \text { and } \\
& Y:=W \backslash B=N_{\bar{G}}(v) \cap W .
\end{aligned}
$$

Note that $X$ and $Y$ are non-empty, since if, for instance, $X=\emptyset$, then $G[U \cup\{v\}]$ and $G[W]$ are complete, so $G$ would be co-bipartite, a contradiction. Moreover, since for every $\left(x^{\prime}, y^{\prime}\right) \in X \times Y$ we have $\left\{x^{\prime}, y^{\prime}\right\} \subseteq X \cup Y \subseteq N_{\bar{G}}(v)$, we must have $\left\{x^{\prime}, y^{\prime}\right\} \in E(G)$, as $\bar{G}$ is triangle-free. Hence, $G[X, Y]$ is complete bipartite.

First suppose that $|Y|$ is even (the case when $|X|$ is even is symmetric). Let $x \in X$ be an arbitrary vertex. In the complete graph $G[Y]$ select a matching $M_{Y}$ on $|Y|$ vertices, and note that $\mathcal{Y}:=\left\{y_{1} y_{2} x: y_{1} y_{2} \in M_{Y}\right\}$ is a triangle packing in $G$ containing $\left|V\left(M_{Y}\right)\right|=|Y|$ edges from $G[U, W]$. Next, let $y \in Y$ be an arbitrary vertex, and in the complete graph $G[X \backslash x]$ select a matching $M_{X}$ on at least $|X|-2$ vertices, so that $\mathcal{X}:=\left\{x_{1} x_{2} y: x_{1} x_{2} \in M_{X}\right\}$ is a triangle packing in $G$ with $\left|V\left(M_{X}\right)\right| \geq|X|-2$ edges from $G[U, W]$. By construction, $\mathcal{X}$ and $\mathcal{Y}$ are edge-disjoint, and $\mathcal{X} \cup \mathcal{Y}$ contains at least $|X|+|Y|-2$ edges from $G[U, W]$.

If both $|X|$ and $|Y|$ are odd, we select $x \in X$ arbitrarily, and $M_{Y}$ to be a matching on $|Y|-1$ vertices. In the second step, we select $y$ to be the sole vertex in $Y \backslash M_{Y}$, and $M_{X}$ to be a matching 
on $|X|-1$ vertices in $X \backslash\{x\}$. We obtain two edge-disjoint triangle packings in $G, \mathcal{X}$ and $\mathcal{Y}$, containing together $|X|+|Y|-2$ edges from $G[U, W]$.

Similarly, in the complete graphs $G[A]$ and $G[B]$ we select matchings $M_{A}$ and $M_{B}$, with at least $|A|-1$ and $|B|-1$ vertices, respectively, to define triangle packings $\mathcal{A}:=\left\{a_{1} a_{2} v: a_{1} a_{2} \in M_{A}\right\}$ and $\mathcal{B}:=\left\{b_{1} b_{2} v: b_{1} b_{2} \in M_{B}\right\}$. Note that $\mathcal{A}$ contains at least $|A|-1$ edges from $G[v, U], \mathcal{B}$ contains at least $|B|-1$ edges from $G[v, W]$, and $\mathcal{A}, \mathcal{B}, \mathcal{X}$ and $\mathcal{Y}$ are edge-disjoint.

Therefore, $\mathcal{A} \cup \mathcal{B} \cup \mathcal{X} \cup \mathcal{Y}$ is a triangle packing of $G$ containing at least

$$
|A|-1+|B|-1+|X|+|Y|-2=|U|+|W|-4=n-5
$$

edges that are not in $G[U]$ or $G[W]$. The edges of $G[U]$ and $G[W]$ that are not part of $\mathcal{A} \cup \mathcal{B} \cup \mathcal{X} \cup \mathcal{Y}$ form on each of $U$ and $W$ a complete graph with a matching removed. Since $\min \{|U|,|W|\} \geq 7$, by Lemma 2.6 those are fractionally decomposable into triangles. Hence,

$$
\begin{aligned}
\nu^{*}(G) & \geq\left(\begin{array}{c}
|U| \\
2
\end{array}\right)+\left(\begin{array}{c}
|W| \\
2
\end{array}\right)+(n-5) \geq 2\left(\begin{array}{c}
\frac{n-1}{2} \\
2
\end{array}\right)+n-5 \\
& =\frac{(n-1)(n-3)+4 n-20}{4}=\frac{n^{2}-17}{4}>\frac{n^{2}-n}{4} .
\end{aligned}
$$

In particular,

$$
\eta(G)=\frac{\nu^{*}(G)}{n(n-1)}>\frac{1}{4}
$$

\section{Proof of Theorem 1.4}

For an $n$-vertex graph $G$ let $\Delta_{\text {bip }}(G)$ denote the edit distance of $G$ to the set of bipartite graphs, i.e. the minimum number of edge deletions needed to turn $G$ into a bipartite graph. Let $E_{b i p}(G):=$ $\Delta_{\text {bip }}(G) / n^{2}$ be the corresponding density. So, $G$ being $\varepsilon$-far from being bipartite is equivalent to $E_{b i p}(G) \geq \varepsilon$.

In order to prove Theorem 1.4, we need the following deep theorem of Alon, Shapira and Sudakov on monotone graph properties ([1], Theorem 1.2), which we state here for the property of being bipartite.

Proposition 4.1. [1] For every $\varepsilon>0$ there is $m(\varepsilon)$ with the following property: let $G$ be any graph and suppose we randomly pick a subset $M$ on $m$ vertices from $V(G)$. Denote by $G^{\prime}$ the graph induced by $G$ on $M$. Then

$$
\operatorname{Prob}\left[\left|E_{b i p}\left(G^{\prime}\right)-E_{b i p}(G)\right|>\varepsilon\right]<\varepsilon .
$$

It is implicit in [1] that $m$ tends to infinity when $\varepsilon$ goes to 0 (in fact, it is not hard to see that this is the only way for Proposition 4.1 to be true). Thus, applying Proposition 4.1 with parameter $\varepsilon / 2$ to graphs $G$ with $E_{b i p}(G) \geq \varepsilon$, we obtain the following statement.

Corollary 4.2. For every $\varepsilon>0$ there exists $m=m(\varepsilon)$, with $m \rightarrow \infty$ as $\varepsilon \rightarrow 0$, as follows. Suppose that $|G|=: n \geq m$, and $G$ is $\varepsilon$-far from being bipartite. Then at least $\left(1-\frac{\varepsilon}{2}\right)\left(\begin{array}{l}n \\ m\end{array}\right) m$-vertex induced subgraphs of $G$ are not bipartite.

Proof of Theorem 1.4. Without loss of generality we may assume that $\varepsilon<1 / 100$. Let $m=m(\varepsilon)$ be as in Corollary 4.2. By choosing $\varepsilon$ to be sufficiently small, by Corollary 4.2 we may assume that $m>100$.

By Lemma 3.1 we have

$$
\min \{\eta(H):|H|=m, \alpha(H) \leq 2, \chi(\bar{H})>2\} \geq \frac{m^{2}-17}{4 m(m-1)}=\frac{1}{4}+\frac{m-17}{4 m(m-1)},
$$


and for co-bipartite graphs $H$ of order $m$, by Lemma 2.4, we have

$$
\eta(H) \geq \frac{m(m-2)}{4 m(m-1)}=\frac{1}{4}-\frac{m}{4 m(m-1)} .
$$

Suppose now that $G$ is co-triangle-free, with $|G|=n \geq m$, and $\bar{G}$ is $\varepsilon$-far from being bipartite. Applying Lemma 2.2 iteratively gives

$$
\eta(G) \geq \frac{1}{\left(\begin{array}{c}
n \\
m
\end{array}\right)} \sum_{M \in\left(\begin{array}{c}
V(G) \\
m
\end{array}\right)} \eta(G[M]) .
$$

Combining this with Corollary 4.2, (4.1) and (4.2), we obtain

$$
\begin{aligned}
\eta(G) & \geq \frac{1}{\left(\begin{array}{c}
n \\
m
\end{array}\right)} \sum_{M \in\left(\begin{array}{c}
V(G) \\
m
\end{array}\right)} \eta(G[M]) \\
& =\frac{1}{\left(\begin{array}{c}
n \\
m
\end{array}\right)}\left(\sum_{M: \chi(\bar{G}[M])>2} \eta(G[M])+\sum_{M: \chi(\bar{G}[M]) \leq 2} \eta(G[M])\right) \\
& \geq\left(1-\frac{\varepsilon}{2}\right)\left(\frac{1}{4}+\frac{m-17}{4 m(m-1)}\right)+\frac{\varepsilon}{2}\left(\frac{1}{4}-\frac{m}{4 m(m-1)}\right) \\
& >\frac{1}{4}+\frac{m-17-\varepsilon m+8 \varepsilon}{4 m(m-1)}>\frac{1}{4}+\frac{1}{8 m} .
\end{aligned}
$$

By the definition of $\eta$ and Proposition 2.1,

$$
\nu(G)>\left(\frac{1}{4}+\frac{1}{8 m}\right) n^{2}+o\left(n^{2}\right) .
$$

Hence, the desired statement holds with $\delta:=1 /(8 m)$.

\section{Discussion}

It suggest itself to use the same approach in order to tackle Conjecture 1.1. Indeed, extending the definition of $\eta$ to arbitrary graphs $G$ via

$$
\eta(G):=\frac{\nu^{*}(G)+\nu^{*}(\bar{G})}{n(n-1)},
$$

the results of Section 2 transfer straightforwardly. That said, for general graphs $\eta(G) \leq 1 / 4$ does not imply that either $G$ or $\bar{G}$ is bipartite. Take, for instance $K_{n / 2, n / 2}$, and add any number $\ell \leq n / 8$ of edges to it. Then the largest monochromatic triangle packing in the resulting colouring $G \cup \bar{G}$ has size at most

$$
2\left(\begin{array}{c}
n / 2 \\
2
\end{array}\right)+2 \ell \leq \frac{n^{2}-2 n}{4}+\frac{n}{4}=\frac{n(n-1)}{4} .
$$

We suspect, however, that this is essentially the only obstruction to having $\eta(G)>1 / 4$. In light of Theorem 1.3, the following strengthening of Conjecture 1.1 appears plausible.

Conjecture 5.1. Suppose that $|G|=n \geq 26$ and $\eta(G) \leq 1 / 4$. Then either $G$ or $\bar{G}$ can be made bipartite by removing at most $n / 8$ edges.

The main challenge in proving Conjecture 5.1 is to bridge the gap between computer simulations for small $n$ and stability arguments for larger $n$. This seems at present much harder for general graphs than in the triangle-free case. 
Further open problems. As several predecessor papers [3, 6] did, we would like to draw the reader's attention to a related conjecture of Jacobson, which states that for every $n$-vertex graph $G$, one of $G$ and $\bar{G}$ will have a triangle packing with at least $n^{2} / 20-o\left(n^{2}\right)$ triangles, which is tight for the $C_{5}$-blowup. To prove this conjecture one would need a new idea, since the averaging approach à la Lemma 2.2 is unlikely to work.

The works [6] and [7] also discussed packings with monochromatic $k$-cliques instead of triangles. It would be interesting to study this systematically for arbitrary fixed graphs $H$, and an arbitrary number of colours.

Question 5.2. For $c \geq 2$ and a fixed graph $H$, how many edge-disjoint monochromatic copies of $H$ are guaranteed to exist in a c-colouring of the edges of $K_{n}$ ?

Specifically, it would be interesting to extend Theorem 1.3 to arbitrary graphs $H$.

Question 5.3. How many edge-disjoint copies of $H$ are guaranteed to exist in an n-vertex graph whose complement is $H$-free?

\section{ACKNOWLEDGEMENT}

I would like to thank David Conlon for helpful discussions, and Olga Goulko for help with setting up the computer simulation.

\section{REFERENCES}

[1] N. Alon, A. Shapira, and B. Sudakov, Additive approximation for edge-deletion problems, Annals of Math 170 (2009), 371-411.

[2] P. Erdős, Some recent problems and results in graph theory, Discrete Math 164 (1997), 81-85.

[3] P. Erdős, R. J. Faudree, R. J. Gould, M. S. Jacobson, and J. Lehel, Edge disjoint monochromatic triangles in 2-colored graphs, Discrete Math 164 (1997), 81-85.

[4] A. W. Goodman, On sets of acquaintances and strangers at any party, Amer. Math. Mon. 66 (1959), 778-783.

[5] P. Haxell and V. Rödl, Integer and fractional packings in dense graphs, Combinatorica 21 (2001), 13-38.

[6] P. Keevash and B. Sudakov, Packing triangles in a graph and its complement, Journal of Graph theory 47(3) (2004), 203-216.

[7] R. Yuster, Packing cliques in graphs with independence number 2, Combin. Probab. Comput. 16(5) (2007), $805-817$.

Department of Mathematics, California Institute of Technology, Pasadena, CA 91125, USA

E-mail address: tyomkyn@caltech.edu 